\title{
On function spaces with fractional Fourier transform in weighted Lebesgue spaces
}

Erdem Toksoy and Ayşe Sandıkçı*

Dedicated to Professor Ravi P Agarwal.

"Correspondence: ayses@omu.edu.tr Department of Mathematics, Faculty of Arts and Sciences, Ondokuz Mayıs University, Samsun, Turkey

\begin{abstract}
Let $w$ and $\omega$ be weight functions on $\mathbb{R}^{d}$. In this work, we define $A_{\alpha, p}^{w, \omega}\left(\mathbb{R}^{d}\right)$ to be the vector space of $f \in L_{w}^{1}\left(\mathbb{R}^{d}\right)$ such that the fractional Fourier transform $F_{\alpha} f$ belongs to $L_{\omega}^{p}\left(\mathbb{R}^{d}\right)$ for $1 \leq p<\infty$. We endow this space with the sum norm $\|f\|_{A_{\alpha, p}^{w, \omega}}=\|f\|_{1, w}+$ $\left\|F_{\alpha} f\right\|_{p, \omega}$ and show that $A_{\alpha, p}^{w, \omega}\left(\mathbb{R}^{d}\right)$ becomes a Banach space and invariant under time-frequency shifts. Further we show that the mapping $y \rightarrow T_{y} f$ is continuous from $\mathbb{R}^{d}$ into $A_{\alpha, p}^{w, \omega}\left(\mathbb{R}^{d}\right)$, the mapping $z \rightarrow M_{z} f$ is continuous from $\mathbb{R}^{d}$ into $A_{\alpha, p}^{w, \omega}\left(\mathbb{R}^{d}\right)$ and $A_{\alpha, p}^{w, \omega}\left(\mathbb{R}^{d}\right)$ is a Banach module over $L_{w}^{1}\left(\mathbb{R}^{d}\right)$ with $\Theta$ convolution operation. At the end of this work, we discuss inclusion properties of these spaces.
\end{abstract}

Keywords: fractional Fourier transform; convolution; Banach module

\section{Introduction}

In this work, for any function $f: \mathbb{R}^{d} \rightarrow \mathbb{C}$, the translation and modulation operator are defined as $T_{x} f(t)=f(t-x)$ and $M_{w} f(t)=e^{i w t} f(t)$ for all $y, w \in \mathbb{R}^{d}$, respectively. Also we write the Lebesgue space $\left(L^{p}\left(\mathbb{R}^{d}\right),\|\cdot\|_{p}\right)$, for $1 \leq p<\infty$. Let $w$ be a weight function on $\mathbb{R}^{d}$, that is, a measurable and locally bounded function $w$ satisfying $w(x) \geq 1$ and $w(x+y) \leq$ $w(x) w(y)$ for all $x, y \in \mathbb{R}^{d}$. We define, for $1 \leq p<\infty$,

$$
L_{w}^{p}\left(\mathbb{R}^{d}\right)=\left\{f \mid f w \in L^{p}\left(\mathbb{R}^{d}\right)\right\} .
$$

It is well known that $L_{w}^{p}\left(\mathbb{R}^{d}\right)$ is a Banach space under the norm $\|f\|_{p, w}=\|f w\|_{p}$.

Let $w_{1}$ and $w_{2}$ are two weight functions. We say that $w_{1} \prec w_{2}$ if there exists $c>0$, such that $w_{1}(x) \leq c w_{2}(x)$ for all $x \in \mathbb{R}^{d}[1,2]$.

The Fourier transform $\hat{f}$ (or $\mathcal{F} f$ ) of $f \in L^{1}(\mathbb{R}$ ) is given by

$$
\hat{f}(w)=\frac{1}{\sqrt{2 \pi}} \int_{-\infty}^{+\infty} f(t) e^{-i w t} d t .
$$

The fractional Fourier transform is a generalization of the Fourier transform with a parameter $\alpha$ and can be interpreted as a rotation by an angle $\alpha$ in the time-frequency plane. The fractional Fourier transform with angle $\alpha$ of a function $f$ is defined by

$$
\mathcal{F}_{\alpha} f(u)=\int_{-\infty}^{+\infty} K_{\alpha}(u, t) f(t) d t
$$

\section{Springer}

o 2015 Toksoy and Sandikcl; licensee Springer. This is an Open Access article distributed under the terms of the Creative Commons Attribution License (http://creativecommons.org/licenses/by/4.0), which permits unrestricted use, distribution, and reproduction in any medium, provided the original work is properly credited. 
where

$$
K_{\alpha}(u, t)= \begin{cases}\sqrt{\frac{1-i \cot \alpha}{2 \pi}} e^{i\left(\frac{u^{2}+t^{2}}{2}\right) \cot \alpha-i u t \operatorname{cosec} \alpha}, & \text { if } \alpha \text { is not multiple of } \pi \\ \delta(t-u), & \text { if } \alpha=2 k \pi, k \in \mathbb{Z}, \\ \delta(t+u), & \text { if } \alpha=(2 k+1) \pi, k \in \mathbb{Z},\end{cases}
$$

and $\delta$ is a Dirac delta function. The fractional Fourier transform with $\alpha=\frac{\pi}{2}$ corresponds to the Fourier transform [3-9].

The fractional Fourier transform can be extended to higher dimensions as [9]:

$$
\begin{aligned}
& \left(\mathcal{F}_{\alpha_{1}, \ldots, \alpha_{n}} f\right)\left(u_{1}, \ldots, u_{n}\right) \\
& \quad=\int_{-\infty}^{+\infty} \cdots \int_{-\infty}^{+\infty} K_{\alpha_{1}, \ldots, \alpha_{n}}\left(u_{1}, \ldots, u_{n} ; t_{1}, \ldots, t_{n}\right) f\left(t_{1}, \ldots, t_{n}\right) d t_{1} \cdots d t_{n}
\end{aligned}
$$

or shortly

$$
\mathcal{F}_{\alpha} f(u)=\int_{-\infty}^{+\infty} \cdots \int_{-\infty}^{+\infty} K_{\alpha}(u, t) f(t) d t
$$

where

$$
K_{\alpha}(u, t)=K_{\alpha_{1}, \ldots, \alpha_{n}}\left(u_{1}, \ldots, u_{n} ; t_{1}, \ldots, t_{n}\right)=K_{\alpha_{1}}\left(u_{1}, t_{1}\right) K_{\alpha_{2}}\left(u_{2}, t_{2}\right) \cdots K_{\alpha_{n}}\left(u_{n}, t_{n}\right) .
$$

In this work we define the function spaces with fractional Fourier transform in weighted Lebesgue spaces and discuss some properties of these spaces.

\section{On function spaces with fractional Fourier transform in weighted Lebesgue spaces}

Definition 1 Let $w$ and $\omega$ be weight functions on $\mathbb{R}^{d}$ and $1 \leq p<\infty$. The space $A_{\alpha, p}^{w, \omega}\left(\mathbb{R}^{d}\right)$ consist of all $f \in L_{w}^{1}\left(\mathbb{R}^{d}\right)$ such that $\mathcal{F}_{\alpha} f \in L_{\omega}^{p}\left(\mathbb{R}^{d}\right)$. The norm on the vector space $A_{\alpha, p}^{w, \omega}\left(\mathbb{R}^{d}\right)$ is

$$
\|f\|_{A_{\alpha, p}^{w, \omega}}=\|f\|_{1, w}+\left\|\mathcal{F}_{\alpha} f\right\|_{p, \omega} .
$$

Theorem $2\left(A_{\alpha, p}^{w, \omega}\left(\mathbb{R}^{d}\right),\|\cdot\|_{A_{\alpha, p}^{w, \omega}}\right)$ is a Banach space for $1 \leq p<\infty$.

Proof Let $\left(f_{n}\right)_{n \in \mathbb{N}}$ is a Cauchy sequence in $A_{\alpha, p}^{w, \omega}\left(\mathbb{R}^{d}\right)$. Thus $\left(f_{n}\right)_{n \in \mathbb{N}}$ and $\left(\mathcal{F}_{\alpha} f_{n}\right)_{n \in \mathbb{N}}$ are Cauchy sequences in $L_{w}^{1}\left(\mathbb{R}^{d}\right)$ and $L_{\omega}^{p}\left(\mathbb{R}^{d}\right)$, respectively. Since $L_{w}^{1}\left(\mathbb{R}^{d}\right)$ and $L_{\omega}^{p}\left(\mathbb{R}^{d}\right)$ are Banach spaces, there exist $f \in L_{w}^{1}\left(\mathbb{R}^{d}\right)$ and $g \in L_{\omega}^{p}\left(\mathbb{R}^{d}\right)$ such that $\left\|f_{n}-f\right\|_{1, w} \rightarrow 0,\left\|\mathcal{F}_{\alpha} f_{n}-g\right\|_{p, \omega} \rightarrow 0$ and hence $\left\|f_{n}-f\right\|_{1} \rightarrow 0$ and $\left\|\mathcal{F}_{\alpha} f_{n}-g\right\|_{p} \rightarrow 0$. Then $\left(\mathcal{F}_{\alpha} f_{n}\right)_{n \in \mathbb{N}}$ has a subsequence $\left(\mathcal{F}_{\alpha} f_{n_{k}}\right)_{n_{k} \in \mathbb{N}}$ that converges pointwise to $g$ almost everywhere. Also it is easy to see that $\left\|f_{n_{k}}-f\right\|_{1} \rightarrow 0$. Then we have

$$
\begin{aligned}
\left|\mathcal{F}_{\alpha} f(u)-g(u)\right| & \leq\left|\mathcal{F}_{\alpha}\left(f_{n_{k}}-f\right)(u)\right|+\left|\mathcal{F}_{\alpha} f_{n_{k}}(u)-g(u)\right| \\
& \leq \prod_{j=1}^{d}\left|\sqrt{\frac{1-i \cot \alpha_{j}}{2 \pi}}\right|
\end{aligned}
$$




$$
\begin{aligned}
& \times \int_{\mathbb{R}^{d}}\left|\left(f_{n_{k}}-f\right)(t)\right|\left|e^{\sum_{j=1}^{d}\left(\frac{i}{2}\left(u_{j}^{2}+t_{j}{ }^{2}\right) \cot \alpha_{j}-i u_{j} t_{j} \operatorname{cosec} \alpha_{j}\right)}\right| d t \\
& +\left|\mathcal{F}_{\alpha} f_{n_{k}}(u)-g(u)\right| \\
= & \prod_{j=1}^{d}\left|\sqrt{\frac{1-i \cot \alpha_{j}}{2 \pi}}\right|\left\|f_{n_{k}}-f\right\|_{1}+\left|\mathcal{F}_{\alpha} f_{n_{k}}(u)-g(u)\right| .
\end{aligned}
$$

From this inequality, we obtain $\mathcal{F}_{\alpha} f=g$ almost everywhere. Thus $\left\|f_{n}-f\right\|_{A_{\alpha, p}^{w, \omega}} \rightarrow 0$ and $f \in A_{\alpha, p}^{w, \omega}\left(\mathbb{R}^{d}\right)$. Hence $\left(A_{\alpha, p}^{w, \omega}\left(\mathbb{R}^{d}\right),\|\cdot\|_{A_{\alpha, p}^{w, \omega}}\right)$ is a Banach space.

The following proposition is generalization of the one-dimensional and two-dimensional versions. The proof of this proposition is very similar to the proofs of onedimensional and two-dimensional versions in $[3,5,10,11]$, and we omit the details.

Proposition 3 Let $\alpha=\left(\alpha_{1}, \alpha_{2}, \ldots, \alpha_{d}\right)$, where $\alpha_{i} \neq k \pi$ for each index $i$ with $1 \leq i \leq d$ and $k \in \mathbb{Z}$. Then

(1) $\mathcal{F}_{\alpha}\left(T_{y} f\right)(u)=e^{\sum_{j=1}^{d}\left(\frac{i}{2} y_{j}^{2} \sin \alpha_{j} \cos \alpha_{j}-i u_{j} y_{j} \sin \alpha_{j}\right)} \mathcal{F}_{\alpha} f\left(u_{1}-y_{1} \cos \alpha_{1}, \ldots, u_{d}-y_{d} \cos \alpha_{d}\right)$

for all $f \in L^{1}\left(\mathbb{R}^{d}\right)$ and $y \in \mathbb{R}^{d}$

(2) $\mathcal{F}_{\alpha}\left(M_{v} f\right)(u)=e^{\sum_{j=1}^{d}\left(-\frac{i}{2} v_{j}^{2} \sin \alpha_{j} \cos \alpha_{j}+i u_{j} v_{j} \cos \alpha_{j}\right)} \mathcal{F}_{\alpha} f\left(u_{1}-v_{1} \sin \alpha_{1}, \ldots, u_{d}-v_{d} \sin \alpha_{d}\right)$

for all $f \in L^{1}\left(\mathbb{R}^{d}\right)$ and $v \in \mathbb{R}^{d}$.

Theorem 4 Let $\alpha=\left(\alpha_{1}, \alpha_{2}, \ldots, \alpha_{d}\right)$, where $\alpha_{i} \neq k \pi$ for each index $i$ with $1 \leq i \leq d$ and $k \in \mathbb{Z}$.

(1) Let $1 \leq p<\infty$, w and $\omega$ be weight functions on $\mathbb{R}^{d}$. Then the space $A_{\alpha, p}^{w, \omega}\left(\mathbb{R}^{d}\right)$ is translation invariant.

(2) Let $\omega$ be a bounded weight function on $\mathbb{R}^{d}$. Then the mapping $y \rightarrow T_{y} f$ of $\mathbb{R}^{d}$ into $A_{\alpha, p}^{w, \omega}\left(\mathbb{R}^{d}\right)$ is continuous.

Proof (1) Let $f \in A_{\alpha, p}^{w, \omega}\left(\mathbb{R}^{d}\right)$. Then $f \in L_{w}^{1}\left(\mathbb{R}^{d}\right)$ and $\mathcal{F}_{\alpha} f \in L_{\omega}^{p}\left(\mathbb{R}^{d}\right)$. It is well known that the space $L_{w}^{1}\left(\mathbb{R}^{d}\right)$ is translation invariant and holds $\left\|T_{x} f\right\|_{1, w} \leq w(y)\|f\|_{1, w}$ for all $y \in \mathbb{R}^{d}$ [12]. Let $b=\left(y_{1} \cos \alpha_{1}, \ldots, y_{d} \cos \alpha_{d}\right)$. By using the equality (1), we get

$$
\begin{aligned}
\left\|\mathcal{F}_{\alpha}\left(T_{y} f\right)\right\|_{p, \omega}= & \left(\int_{\mathbb{R}^{d}}\left|\mathcal{F}_{\alpha}\left(T_{y} f\right)(u)\right|^{p} \omega^{p}(u) d u\right)^{\frac{1}{p}} \\
= & \left(\int_{\mathbb{R}^{d}}\left|\mathcal{F}_{\alpha} f\left(u_{1}-y_{1} \cos \alpha_{1}, \ldots, u_{d}-y_{d} \cos \alpha_{d}\right)\right|^{p}\right. \\
& \left.\times\left|e^{\sum_{j=1}^{d}\left(\frac{i}{2} y_{j}{ }^{2} \sin \alpha_{j} \cos \alpha_{j}-i u_{j} y_{j} \sin \alpha_{j}\right)}\right|^{p} \omega^{p}(u) d u\right)^{\frac{1}{p}} \\
\leq & \left(\int_{\mathbb{R}^{d}}\left|\mathcal{F}_{\alpha} f(u-b)\right|^{p} \omega^{p}(u-b) \omega^{p}(b) d u\right)^{\frac{1}{p}} \\
= & \omega(b)\left\|\mathcal{F}_{\alpha} f\right\|_{p, \omega}
\end{aligned}
$$


for all $y \in \mathbb{R}^{d}$. Hence, we have

$$
\left\|T_{y} f\right\|_{A_{\alpha, p}^{w, \omega}} \leq w(y)\|f\|_{1, w}+\omega(b)\left\|\mathcal{F}_{\alpha} f\right\|_{p, \omega}<\infty
$$

This means that $A_{\alpha, p}^{w, \omega}\left(\mathbb{R}^{d}\right)$ is translation invariant.

(2) Let $f \in A_{\alpha, p}^{w, \omega}\left(\mathbb{R}^{d}\right)$. We will show that if $\lim _{n \rightarrow \infty} y_{n}=0$ for any sequence $\left(y_{n}\right)_{n \in \mathbb{N}} \subset \mathbb{R}^{d}$, then $\lim _{n \rightarrow \infty} T_{y_{n}} f=f$, which will complete the proof. It is well known that the mapping $y \rightarrow T_{y} f$ is continuous from $\mathbb{R}^{d}$ into $L_{w}^{1}\left(\mathbb{R}^{d}\right)$ (see [12]). Thus, we have

$$
\left\|T_{y_{n}} f-f\right\|_{1, w} \rightarrow 0
$$

as $n \rightarrow \infty$. Also,

$$
\begin{aligned}
\left\|\mathcal{F}_{\alpha}\left(T_{y_{n}} f-f\right)\right\|_{p, \omega}= & \left\|\mathcal{F}_{\alpha}\left(T_{y_{n}} f\right)-\mathcal{F}_{\alpha} f\right\|_{p, \omega} \\
= & \left\|e^{\sum_{j=1}^{d}\left(\frac{i}{2}\left(y_{n}^{\prime}\right)^{2} \sin \alpha_{j} \cos \alpha_{j}-i u_{j} y_{n}^{j} \sin \alpha_{j}\right)} T_{\left(y_{n}^{1} \cos \alpha_{1}, \ldots, y_{n}^{d} \cos \alpha_{d}\right)}\left(\mathcal{F}_{\alpha} f\right)-\mathcal{F}_{\alpha} f\right\|_{p, \omega} \\
\leq & \left\|\left(T_{\left(y_{n}^{1} \cos \alpha_{1}, \ldots, y_{n}^{d} \cos \alpha_{d}\right)}\left(\mathcal{F}_{\alpha} f\right)-\mathcal{F}_{\alpha} f\right)\right\|_{p, \omega} \\
& +\left\|\left(e^{\sum_{j=1}^{d}\left(\frac{i}{2}\left(y_{n}^{j}\right)^{2} \sin \alpha_{j} \cos \alpha_{j}-i u_{j} y_{n}^{j} \sin \alpha_{j}\right)}-1\right) \mathcal{F}_{\alpha} f\right\|_{p, \omega}
\end{aligned}
$$

Since $\mathcal{F}_{\alpha} f \in L_{\omega}^{p}\left(\mathbb{R}^{d}\right)$, the mapping $y \rightarrow T_{y}\left(\mathcal{F}_{\alpha} f\right)$ is continuous from $\mathbb{R}^{d}$ into $L_{\omega}^{p}\left(\mathbb{R}^{d}\right)$ for all $y \in \mathbb{R}^{d}$ [12]. Then we obtain $\left\|T_{\left(y_{n}^{1} \cos \alpha_{1}, \ldots, y_{n}^{d} \cos \alpha_{d}\right)}\left(\mathcal{F}_{\alpha} f\right)-\mathcal{F}_{\alpha} f\right\|_{p, \omega} \rightarrow 0$ as $n \rightarrow \infty$. Now let $h_{y_{n}}(u)=\left|e^{\sum_{j=1}^{d}\left(\frac{i}{2}\left(y_{n}^{j}\right)^{2} \sin \alpha_{j} \cos \alpha_{j}-i u_{j} y_{n}^{j} \sin \alpha_{j}\right)}-1\right|\left|\mathcal{F}_{\alpha} f(u)\right|$. Since $\lim _{n \rightarrow \infty} y_{n}=0$ and $\omega$ is a bounded weight function on $\mathbb{R}^{d}$, we see that $\lim _{n \rightarrow \infty} h_{y_{n}}^{p}(u) \omega^{p}(u)=0$ for all $u \in \mathbb{R}^{d}$. Also, since

$$
h_{y_{n}}(u)=\left|e^{\sum_{j=1}^{d}\left(\frac{i}{2}\left(y_{n}^{j}\right)^{2} \sin \alpha_{j} \cos \alpha_{j}-i u_{j} y_{n} \sin \alpha_{j}\right)}-1\right|\left|\mathcal{F}_{\alpha} f(u)\right| \leq 2\left|\mathcal{F}_{\alpha} f(u)\right|
$$

and $\mathcal{F}_{\alpha} f \in L_{\omega}^{p}\left(\mathbb{R}^{d}\right)$, we can write $h_{y_{n}}^{p}(u) \omega^{p}(u) \leq 2^{p}\left|\mathcal{F}_{\alpha} f(u)\right|^{p} \omega^{p}(u)$. Thus, by the Lebesgue dominated convergence theorem,

$$
\left\|\left(e^{\sum_{j=1}^{d}\left(\frac{i}{2}\left(y_{n}^{\prime}\right)^{2} \sin \alpha_{j} \cos \alpha_{j}-i u_{j} y_{n}^{j} \sin \alpha_{j}\right)}-1\right) \mathcal{F}_{\alpha} f\right\|_{p, \omega} \rightarrow 0
$$

as $\lim _{n \rightarrow \infty} y_{n}=0$. Hence,

$$
\left\|T_{y_{n}} f-f\right\|_{A_{\alpha, p}^{w, \omega}} \rightarrow 0
$$

as $n \rightarrow \infty$. Combining (2) and (3),

$$
\left\|T_{y_{n}} f-f\right\|_{A_{\alpha, p}^{w, \omega}}=\left\|T_{y_{n}} f-f\right\|_{1, w}+\left\|\mathcal{F}_{\alpha}\left(T_{y_{n}} f-f\right)\right\|_{p, \omega} \rightarrow 0
$$

as $n \rightarrow \infty$. This is the desired result.

Theorem 5 Let $\alpha=\left(\alpha_{1}, \alpha_{2}, \ldots, \alpha_{d}\right)$, where $\alpha_{i} \neq k \pi$ for each index $i$ with $1 \leq i \leq d$ and $k \in \mathbb{Z}$. 
(1) Let $1 \leq p<\infty$, w and $\omega$ be weight functions on $\mathbb{R}^{d}$. Then $A_{\alpha, p}^{w, \omega}\left(\mathbb{R}^{d}\right)$ is invariant under modulations.

(2) Let $\omega$ be a bounded weight function on $\mathbb{R}^{d}$. Then the mapping $z \rightarrow M_{z} f$ is continuous from $\mathbb{R}^{d}$ into $A_{\alpha, p}^{w, \omega}\left(\mathbb{R}^{d}\right)$.

Proof (1) Let $f \in A_{\alpha, p}^{w, \omega}\left(\mathbb{R}^{d}\right)$. Then $f \in L_{w}^{1}\left(\mathbb{R}^{d}\right)$ and $\mathcal{F}_{\alpha} f \in L_{\omega}^{p}\left(\mathbb{R}^{d}\right)$. It is easy to see that $\left\|M_{\eta} f\right\|_{1, w}=\|f\|_{1, w}$ and $M_{\eta} f \in L_{w}^{1}\left(\mathbb{R}^{d}\right)$. Let $c=\left(\eta_{1} \sin \alpha_{1}, \ldots, \eta_{d} \sin \alpha_{d}\right) \in \mathbb{R}^{d}$. Thus by Proposition 3 , we have

$$
\begin{aligned}
\left\|\mathcal{F}_{\alpha}\left(M_{\eta} f\right)\right\|_{p, \omega}= & \left(\int_{\mathbb{R}^{d}}\left|\mathcal{F}_{\alpha}\left(M_{\eta} f\right)(u)\right|^{p} \omega^{p}(u) d u\right)^{\frac{1}{p}} \\
= & \left(\int_{\mathbb{R}^{d}}\left|\mathcal{F}_{\alpha} f\left(u_{1}-\eta_{1} \sin \alpha_{1}, \ldots, u_{d}-\eta_{d} \sin \alpha_{d}\right)\right|^{p}\right. \\
& \left.\times\left|e^{\sum_{j=1}^{d}\left(-\frac{i}{2} \eta_{j}^{2} \sin \alpha_{j} \cos \alpha_{j}+i u_{j} \eta_{j} \cos \alpha_{j}\right)}\right|^{p} \omega^{p}(u) d u\right)^{\frac{1}{p}} \\
\leq & \left(\int_{\mathbb{R}^{d}}\left|\mathcal{F}_{\alpha} f(u-c)\right|^{p} \omega^{p}(u-c) \omega^{p}(c) d u\right)^{\frac{1}{p}} \\
= & \omega(c)\left\|\mathcal{F}_{\alpha} f\right\|_{p, \omega}
\end{aligned}
$$

for all $\eta \in \mathbb{R}^{d}$. Hence, we get

$$
\left\|M_{\eta} f\right\|_{A_{\alpha, p}^{w, \omega}} \leq\|f\|_{1, w}+\omega(c)\left\|\mathcal{F}_{\alpha} f\right\|_{p, \omega}<\infty
$$

(2) The proof technique of this part is the same as that of Theorem 4(2). So, for the sake of brevity, we will not prove it.

The following definition is an extension of the convolution in $[13,14]$ of two functions to $n$ dimensions.

Definition 6 Let $\alpha=\left(\alpha_{1}, \alpha_{2}, \ldots, \alpha_{d}\right)$, where $\alpha_{i} \neq k \pi$ for each index $i$ with $1 \leq i \leq d$ and $k \in \mathbb{Z}$. Then the convolution of two functions $f, g \in L^{1}\left(\mathbb{R}^{d}\right)$ is the function $f \Theta g$ defined by

$$
(f \Theta g)(x)=\int_{\mathbb{R}^{d}} f(y) g(x-y) e^{\sum_{j=1}^{d} i y_{j}\left(y_{j}-x_{j}\right) \cot \alpha_{j}} d y .
$$

It is easy to see that $f \Theta g$ belongs to $L^{1}\left(\mathbb{R}^{d}\right)$ by Fubini's theorem.

Theorem 7 Let $\alpha=\left(\alpha_{1}, \alpha_{2}, \ldots, \alpha_{d}\right)$, where $\alpha_{i} \neq k \pi$ for each index $i$ with $1 \leq i \leq d$ and $k \in \mathbb{Z}$, and $f, g \in L^{1}\left(\mathbb{R}^{d}\right)$. Then

$$
\mathcal{F}_{\alpha}(f \Theta g)(u)=\left[\prod_{j=1}^{d} \sqrt{\frac{2 \pi}{1-i \cot \alpha_{j}}}\right] e^{\sum_{j=1}^{d}-\frac{i}{2} u_{j}^{2} \cot \alpha_{j}} \mathcal{F}_{\alpha} f(u) \mathcal{F}_{\alpha} g(u),
$$

where $\mathcal{F}_{\alpha} f$ and $\mathcal{F}_{\alpha} g$ are the fractional Fourier transforms of functions $f$ and $g$, respectively. 
Proof Let $\alpha=\left(\alpha_{1}, \alpha_{2}, \ldots, \alpha_{d}\right)$, where $\alpha_{i} \neq k \pi$ for each index $i$ with $1 \leq i \leq d$ and $k \in \mathbb{Z}$, and $f, g \in L^{1}\left(\mathbb{R}^{d}\right)$. We can write from the definition of the fractional Fourier transform

$$
\begin{aligned}
\mathcal{F}_{\alpha}(f \Theta g)(u)= & {\left[\prod_{j=1}^{d} \sqrt{\frac{1-i \cot \alpha_{j}}{2 \pi}}\right] \int_{\mathbb{R}^{d}}(f \Theta g)(t) e^{\sum_{j=1}^{d}\left(\frac{i}{2}\left(u_{j}^{2}+t_{j}^{2}\right) \cot \alpha_{j}-i u_{j} t_{j} \operatorname{cosec} \alpha_{j}\right)} d t } \\
= & {\left[\prod_{j=1}^{d} \sqrt{\frac{1-i \cot \alpha_{j}}{2 \pi}}\right] \int_{\mathbb{R}^{d}} \int_{\mathbb{R}^{d}} f(y) g(t-y) e^{\sum_{j=1}^{d} i y_{j}\left(y_{j}-t_{j}\right) \cot \alpha_{j}} } \\
& \times e^{\sum_{j=1}^{d}\left(\frac{i}{2}\left(u_{j}^{2}+t_{j}^{2}\right) \cot \alpha_{j}-i u_{j} t_{j} \operatorname{cosec} \alpha_{j}\right)} d t d y .
\end{aligned}
$$

We make the substitution $t-y=k$ and obtain

$$
\begin{aligned}
\mathcal{F}_{\alpha}(f \Theta g)(u)= & {\left[\prod_{j=1}^{d} \sqrt{\frac{1-i \cot \alpha_{j}}{2 \pi}}\right] \int_{\mathbb{R}^{d}}\left(\int_{\mathbb{R}^{d}} f(y) e^{\sum_{j=1}^{d}\left(\frac{i}{2}\left(u_{j}^{2}+y_{j}^{2}\right) \cot \alpha_{j}-i u_{j} y_{j} \operatorname{cosec} \alpha_{j}\right)} d y\right) } \\
& \times g(k) e^{\sum_{j=1}^{d}\left(\frac{i}{2} k_{j}^{2} \cot \alpha_{j}-i u_{j} k_{j} \operatorname{cosec} \alpha_{j}\right)} d k \\
= & {\left[\prod_{j=1}^{d} \sqrt{\frac{2 \pi}{1-i \cot \alpha_{j}}}\right] e^{\sum_{j=1}^{d}-\frac{i}{2} u_{j}^{2} \cot \alpha_{j}}\left[\prod_{j=1}^{d} \sqrt{\frac{1-i \cot \alpha_{j}}{2 \pi}}\right]^{2} } \\
& \times \int_{\mathbb{R}^{d}}\left(\int_{\mathbb{R}^{d}} f(y) e^{\sum_{j=1}^{d}\left(\frac{i}{2}\left(u_{j}^{2}+y_{j}^{2}\right) \cot \alpha_{j}-i u_{j} y_{j} \operatorname{cosec} \alpha_{j}\right)} d y\right) \\
& \times g(k) e^{\sum_{j=1}^{d}\left(\frac{i}{2}\left(k_{j}^{2}+u_{j}^{2}\right) \cot \alpha_{j}-i u_{j} k_{j} \operatorname{cosec} \alpha_{j}\right)} d k \\
= & {\left[\prod_{j=1}^{d} \sqrt{\frac{2 \pi}{1-i \cot \alpha_{j}}}\right] e^{\sum_{j=1}^{d}-\frac{i}{2} u_{j}^{2} \cot \alpha_{j}}\left[\prod_{j=1}^{d} \sqrt{\frac{1-i \cot \alpha_{j}}{2 \pi}}\right] } \\
& \times \int_{\mathbb{R}^{d}} \mathcal{F}_{\alpha} f(u) g(k) e^{\sum_{j=1}^{d}\left(\frac{i}{2}\left(k_{j}^{2}+u_{j}^{2}\right) \cot \alpha_{j}-i u_{j} k_{j} \operatorname{cosec} \alpha_{j}\right)} d k \\
= & {\left[\prod_{j=1}^{d} \sqrt{\frac{2 \pi}{1-i \cot \alpha_{j}}}\right] e^{\sum_{j=1}^{d}-\frac{i}{2} u_{j}^{2} \cot \alpha_{j}} \mathcal{F}_{\alpha} f(u) \mathcal{F}_{\alpha} g(u) . }
\end{aligned}
$$

Theorem 8 Let $\alpha=\left(\alpha_{1}, \alpha_{2}, \ldots, \alpha_{d}\right)$, where $\alpha_{i} \neq k \pi$ for each index $i$ with $1 \leq i \leq d$ and $k \in \mathbb{Z}$. $L_{w}^{1}\left(\mathbb{R}^{d}\right)$ is a Banach algebra under $\Theta$ convolution.

Proof It is well known that $L_{w}^{1}\left(\mathbb{R}^{d}\right)$ is a Banach space [2]. Let $f, g \in L_{w}^{1}\left(\mathbb{R}^{d}\right)$, then we have

$$
\begin{aligned}
\|f \Theta g\|_{1, w} & =\int_{\mathbb{R}^{d}}|f \Theta g| w(x) d y \\
& =\int_{\mathbb{R}^{d}}\left|\int_{\mathbb{R}^{d}} f(y) g(x-y) e^{\sum_{j=1}^{d} i y_{j}\left(y_{j}-x_{j}\right) \cot \alpha_{j}} d y\right| w(x) d x \\
& \leq \int_{\mathbb{R}^{d}}\left(\int_{\mathbb{R}^{d}}|g(x-y)| w(x-y) d x\right)|f(y)| w(y) d y \\
& =\|g\|_{1, w} \int_{\mathbb{R}^{d}}|f(y)| w(y) d y \\
& =\|g\|_{1, w}\|f\|_{1, w} .
\end{aligned}
$$

It is easy to show that the other conditions of the Banach algebra are satisfied. 
Theorem 9 Let $\alpha=\left(\alpha_{1}, \alpha_{2}, \ldots, \alpha_{d}\right)$, where $\alpha_{i} \neq k \pi$ for each index $i$ with $1 \leq i \leq d$ and $k \in \mathbb{Z}$. $A_{\alpha, p}^{w, \omega}\left(\mathbb{R}^{d}\right)$ is a Banach $\Theta$-convolution module over $L_{w}^{1}\left(\mathbb{R}^{d}\right)$.

Proof It is well known that $A_{\alpha, p}^{w, \omega}\left(\mathbb{R}^{d}\right)$ is a Banach space by Theorem 2. Let $f \in A_{\alpha, p}^{w, \omega}\left(\mathbb{R}^{d}\right)$ and $g \in L_{w}^{1}\left(\mathbb{R}^{d}\right)$. By using the inequality (4), we get

$$
\begin{aligned}
\left\|\mathcal{F}_{\alpha}(f \Theta g)\right\|_{p, \omega}= & \left\|\left[\prod_{j=1}^{d} \sqrt{\frac{2 \pi}{1-i \cot \alpha_{j}}}\right] e^{\sum_{j=1}^{d}-\frac{i}{2} u_{j}^{2} \cot \alpha_{j}} \mathcal{F}_{\alpha} f(u) \mathcal{F}_{\alpha} g(u)\right\|_{p, \omega} \\
= & \left|\prod_{j=1}^{d} \sqrt{\frac{2 \pi}{1-i \cot \alpha_{j}}}\right|\left(\int_{\mathbb{R}^{d}}\left|\mathcal{F}_{\alpha} f(u)\right|^{p}\left|\mathcal{F}_{\alpha} g(u)\right|^{p} \omega^{p}(u) d u\right)^{\frac{1}{p}} \\
= & \left|\prod_{j=1}^{d} \sqrt{\frac{2 \pi}{1-i \cot \alpha_{j}}}\right|\left(\int_{\mathbb{R}^{d}}\left|\mathcal{F}_{\alpha} f(u)\right|^{p}\left|\prod_{j=1}^{d} \sqrt{\frac{1-i \cot \alpha_{j}}{2 \pi}}\right|^{p}\right. \\
& \left.\times\left|\int_{\mathbb{R}^{d}} g(t) e^{\sum_{j=1}^{d}\left(\frac{i}{2}\left(u_{j}^{2}+t_{j}^{2}\right) \cot \alpha_{j}-i u_{j} t_{j} \operatorname{cosec} \alpha_{j}\right)} d t\right|^{p} \omega^{p}(u) d u\right)^{\frac{1}{p}} \\
\leq & \left(\int_{\mathbb{R}^{d}}\left|\mathcal{F}_{\alpha} f(u)\right|^{p}\left(\int_{\mathbb{R}^{d}}|g(t)| d t\right)^{p} \omega^{p}(u) d u\right)^{\frac{1}{p}} \\
= & \|g\|_{1}\left(\int_{\mathbb{R}^{d}}\left|\mathcal{F}_{\alpha} f(u)\right|^{p} \omega^{p}(u) d u\right)^{\frac{1}{p}} \\
\leq & \|g\|_{1, w}\left\|\mathcal{F}_{\alpha} f\right\|_{p, \omega} .
\end{aligned}
$$

Combining (4) and (5), we obtain

$$
\begin{aligned}
\|f \Theta g\|_{A_{\alpha, p}^{w, \omega}} & =\|f \Theta g\|_{1, w}+\left\|\mathcal{F}_{\alpha}(f \Theta g)\right\|_{p, \omega} \\
& \leq\|g\|_{1, w}\|f\|_{1, w}+\|g\|_{1, w}\left\|\mathcal{F}_{\alpha} f\right\|_{p, \omega} \\
& =\|f\|_{A_{\alpha, p}^{w, \omega}}\|g\|_{1, w} .
\end{aligned}
$$

This is the desired result. It is easy to see that the other conditions of the module are satisfied.

\section{Inclusion properties of the space $A_{\alpha, p}^{w, \omega}\left(\mathbb{R}^{d}\right)$}

Proposition 10 For every $0 \neq f \in A_{\alpha, p}^{w, 1}\left(\mathbb{R}^{d}\right)$ there exists $c(f)>0$ such that

$$
c(f) w(x) \leq\left\|T_{x} f\right\|_{A_{\alpha, p}^{w, 1}} \leq w(x)\|f\|_{A_{\alpha, p}^{w, p}}
$$

Proof Let $0 \neq f \in A_{\alpha, p}^{w, 1}\left(\mathbb{R}^{d}\right)$. By [12], there exists $c(f)>0$ such that

$$
c(f) w(x) \leq\left\|T_{x} f\right\|_{1, w} \leq w(x)\|f\|_{1, w} .
$$

By using (6) and the equality $\left\|\mathcal{F}_{\alpha}\left(T_{x} f\right)\right\|_{p}=\left\|\mathcal{F}_{\alpha} f\right\|_{p}$, we obtain

$$
\begin{aligned}
c(f) w(x) & \leq\left\|T_{x} f\right\|_{1, w} \leq\left\|T_{x} f\right\|_{1, w}+\left\|\mathcal{F}_{\alpha}\left(T_{x} f\right)\right\|_{p} \\
& \leq w(x)\|f\|_{1, w}+\left\|\mathcal{F}_{\alpha} f\right\|_{p}
\end{aligned}
$$




$$
\begin{aligned}
& \leq w(x)\|f\|_{1, w}+w(x)\left\|\mathcal{F}_{\alpha} f\right\|_{p} \\
& =w(x)\|f\|_{A_{\alpha, p}^{w, 1}}
\end{aligned}
$$

for all $f \in A_{\alpha, p}^{w, 1}\left(\mathbb{R}^{d}\right)$.

Lemma 11 Let $w_{1}, w_{2}, \omega_{1}$ and $\omega_{2}$ be weight functions on $\mathbb{R}^{d}$. If $A_{\alpha, p}^{w_{1}, \omega_{1}}\left(\mathbb{R}^{d}\right) \subset A_{\alpha, p}^{w_{2}, \omega_{2}}\left(\mathbb{R}^{d}\right)$, then $A_{\alpha, p}^{w_{1}, \omega_{1}}\left(\mathbb{R}^{d}\right)$ is a Banach space under the norm $\|f\|=\|f\|_{A_{\alpha, p}^{w_{1}, \omega_{1}}}+\|f\|_{A_{\alpha, p}^{w_{2}, \omega_{2}}}$.

Proof Let $\left(f_{n}\right)_{n \in \mathbb{N}}$ is a Cauchy sequence in $\left(A_{\alpha, p}^{w_{1}, \omega_{1}}\left(\mathbb{R}^{d}\right),\|\| \cdot \| \mid\right)$. Then $\left(f_{n}\right)_{n \in \mathbb{N}}$ is a Cauchy sequence in $\left(A_{\alpha, p}^{w_{1}, \omega_{1}}\left(\mathbb{R}^{d}\right),\|\cdot\|_{A_{\alpha, p}^{w_{1}, \omega_{1}}}\right)$ and $\left(A_{\alpha, p}^{w_{2}, \omega_{2}}\left(\mathbb{R}^{d}\right),\|\cdot\|_{A_{\alpha, p}^{w_{2}, \omega_{2}}}\right)$. As these spaces are Banach spaces, there exist $f \in A_{\alpha, p}^{w_{1}, \omega_{1}}\left(\mathbb{R}^{d}\right)$ and $g \in A_{\alpha, p}^{w_{2}, \omega_{2}}\left(\mathbb{R}^{d}\right)$ such that $\left\|f_{n}-f\right\|_{A_{\alpha, p}^{w_{1}, \omega_{1}}} \rightarrow$ $0,\left\|f_{n}-g\right\|_{A_{\alpha, p}^{w_{2}, \omega_{2}}} \rightarrow 0$. Using the inequalities $\|\cdot\|_{1} \leq\|\cdot\|_{1, w_{1}} \leq\|\cdot\|_{A_{\alpha, p}^{w_{1}, \omega_{1}}}$ and $\|\cdot\|_{1} \leq$ $\|\cdot\|_{1, w_{2}} \leq\|\cdot\|_{A_{\alpha, p}^{w_{2}, \omega_{2}}}$, we obtain $\left\|f_{n}-f\right\|_{1} \rightarrow 0$ and $\left\|f_{n}-g\right\|_{1} \rightarrow 0$. Also $\|f-g\|_{1} \leq\left\|f_{n}-f\right\|_{1}+$ $\left\|f_{n}-g\right\|_{1}$, we have $f=g$. Hence $\left\|f_{n}-f\right\| \rightarrow 0$ and $f \in A_{\alpha, p}^{w_{1}, \omega_{1}}\left(\mathbb{R}^{d}\right)$. That means $\left(A_{\alpha, p}^{w_{1}, \omega_{1}}\left(\mathbb{R}^{d}\right)\right.$, $\||\cdot| \mid)$ is a Banach space.

Theorem 12 Let $w_{1}$ and $w_{2}$ be weight functions on $\mathbb{R}^{d}$. Then $A_{\alpha, p}^{w_{1}, 1}\left(\mathbb{R}^{d}\right) \subset A_{\alpha, p}^{w_{2}, 1}\left(\mathbb{R}^{d}\right)$ if and only if $w_{2} \prec w_{1}$.

Proof Suppose that $w_{2} \prec w_{1}$. Thus there exists $c_{1}>0$ such that $w_{2}(x) \leq c_{1} w_{1}(x)$ for all $x \in$ $\mathbb{R}^{d}$. Also let $f \in A_{\alpha, p}^{w_{1}, 1}\left(\mathbb{R}^{d}\right)$. Then we write

$$
\|f\|_{1, w_{2}} \leq c_{1}\|f\|_{1, w_{1}}<\infty
$$

Hence we have

$$
\|f\|_{A_{\alpha, p}^{w_{2}, 1}}=\|f\|_{1, w_{2}}+\left\|\mathcal{F}_{\alpha} f\right\|_{p} \leq c_{1}\|f\|_{1, w_{1}}+c_{1}\left\|\mathcal{F}_{\alpha} f\right\|_{p}=c_{1}\|f\|_{A_{\alpha, p}^{w_{1}, 1}}
$$

Therefore, $A_{\alpha, p}^{w_{1}, 1}\left(\mathbb{R}^{d}\right) \subset A_{\alpha, p}^{w_{2}, 1}\left(\mathbb{R}^{d}\right)$.

Conversely, suppose that $A_{\alpha, p}^{w_{1}, 1}\left(\mathbb{R}^{d}\right) \subset A_{\alpha, p}^{w_{2}, 1}\left(\mathbb{R}^{d}\right)$. For every $f \in A_{\alpha, p}^{w_{1}, 1}\left(\mathbb{R}^{d}\right)$, we have $f \in$ $A_{\alpha, p}^{w_{2}, 1}\left(\mathbb{R}^{d}\right)$. By Proposition 10 , there are constants $c_{1}, c_{2}, c_{3}, c_{4}>0$ such that

$$
c_{1} w_{1}(x) \leq\left\|T_{x} f\right\|_{A_{\alpha, p}^{w_{1}, 1}} \leq c_{2} w_{1}(x)
$$

and

$$
c_{3} w_{2}(x) \leq\left\|T_{x} f\right\|_{A_{\alpha, p}^{w_{2}, 1}} \leq c_{4} w_{2}(x)
$$

for all $x \in \mathbb{R}^{d}$. It is well known from Lemma 11 that the space $A_{\alpha, p}^{w_{1}, 1}\left(\mathbb{R}^{d}\right)$ is a Banach space under the norm $\|f\|, f \in A_{\alpha, p}^{w_{1}, 1}\left(\mathbb{R}^{d}\right)$. Then by the closed graph theorem the norms $\|\cdot\|_{A_{\alpha, p}^{w_{1}, 1}}$ and $\|\cdot\|_{A_{\alpha, p}^{w_{2}, 1}}$ are equivalent on $A_{\alpha, p}^{w_{1}, 1}\left(\mathbb{R}^{d}\right)$. So, there exists $c>0$ such that $\|f\|_{A_{\alpha, p}^{w_{2}, 1}} \leq\|f\|_{A_{\alpha, p}^{w_{1}, 1}}$ for all $f \in A_{\alpha, p}^{w_{1}, 1}\left(\mathbb{R}^{d}\right)$. Moreover, as $T_{x} f \in A_{\alpha, p}^{w_{2}, 1}\left(\mathbb{R}^{d}\right)$, we have

$$
\left\|T_{x} f\right\|_{A_{\alpha, p}^{w_{2}, 1}} \leq c\left\|T_{x} f\right\|_{A_{\alpha, p}^{w_{1}, 1}}
$$


Then, combining (7), (8), and (9), we obtain

$$
c_{3} w_{2}(x) \leq\left\|T_{x} f\right\|_{A_{\alpha, p}^{w_{2}, 1}} \leq c\left\|T_{x} f\right\|_{A_{\alpha, p}^{w_{1}, 1}} \leq c c_{2} w_{1}(x) .
$$

Thus, $w_{2}(x) \leq \frac{c c_{2}}{c_{3}} w_{1}(x)$. Let $\frac{c c_{2}}{c_{3}}=k$. Then we find $w_{2}(x) \leq k w_{1}(x)$ for all $x \in \mathbb{R}^{d}$.

Proposition 13 Let $w_{1}, w_{2}, \omega_{1}$ and $\omega_{2}$ be weight functions on $\mathbb{R}^{d}$. If $w_{2} \prec w_{1}$ and $\omega_{2} \prec \omega_{1}$, then $A_{\alpha, p}^{w_{1}, \omega_{1}}\left(\mathbb{R}^{d}\right) \subset A_{\alpha, p}^{w_{2}, \omega_{2}}\left(\mathbb{R}^{d}\right)$.

Proof Assume that $w_{2} \prec w_{1}$ and $\omega_{2} \prec \omega_{1}$. Then there exist $c_{1}, c_{2}>0$ such that $w_{2}(x) \leq$ $c_{1} w_{1}(x)$ and $\omega_{2}(x) \leq c_{2} \omega_{1}(x)$ for all $x \in \mathbb{R}^{d}$. Let $f \in A_{\alpha, p}^{w_{1}, \omega_{1}}\left(\mathbb{R}^{d}\right)$. As $f \in L_{w_{1}}^{1}\left(\mathbb{R}^{d}\right)$ and $\mathcal{F}_{\alpha} f \in$ $L_{\omega_{1}}^{p}\left(\mathbb{R}^{d}\right)$, we have $\|f\|_{1, w_{2}} \leq c_{1}\|f\|_{1, w_{1}}<\infty$ and $\left\|\mathcal{F}_{\alpha} f\right\|_{p, \omega_{2}} \leq c_{2}\left\|\mathcal{F}_{\alpha} f\right\|_{p, \omega_{1}}<\infty$. Hence, we obtain $f \in A_{\alpha, p}^{w_{2}, \omega_{2}}\left(\mathbb{R}^{d}\right)$, and then $A_{\alpha, p}^{w_{1}, \omega_{1}}\left(\mathbb{R}^{d}\right) \subset A_{\alpha, p}^{w_{2}, \omega_{2}}\left(\mathbb{R}^{d}\right)$.

\section{Duality}

Let the mapping $\Phi: A_{\alpha, p}^{w, \omega}\left(\mathbb{R}^{d}\right) \rightarrow L_{w}^{1}\left(\mathbb{R}^{d}\right) \times L_{\omega}^{p}\left(\mathbb{R}^{d}\right)$ be defined by $\Phi(f)=\left(f, \mathcal{F}_{\alpha} f\right)$ for $1 \leq$ $p<\infty$ and let $H=\Phi\left(A_{\alpha, p}^{w, \omega}\left(\mathbb{R}^{d}\right)\right)$. Then

$$
\|\Phi(f)\|=\left\|\left(f, \mathcal{F}_{\alpha} f\right)\right\|=\|f\|_{1, w}+\left\|\mathcal{F}_{\alpha} f\right\|_{p, \omega}
$$

is a norm on $H$ for all $f \in A_{\alpha, p}^{w, \omega}\left(\mathbb{R}^{d}\right)$. Moreover, we define a set $K$ as

$$
\begin{aligned}
K= & \left\{(\varphi, \psi):\left((\varphi, \psi) \in L_{w^{-1}}^{\infty}\left(\mathbb{R}^{d}\right) \times L_{\omega^{-1}}^{p^{\prime}}\left(\mathbb{R}^{d}\right)\right),\right. \\
& \left.\int_{\mathbb{R}^{d}} f(x) \varphi(x) d x+\int_{\mathbb{R}^{d}} \mathcal{F}_{\alpha} f(y) \psi(y) d y=0 \text { for all }\left(f, \mathcal{F}_{\alpha} f\right) \in H\right\},
\end{aligned}
$$

where $\frac{1}{p}+\frac{1}{p^{\prime}}=1$.

The following proposition is proved by the duality theorem, Theorem 1.7 in [15].

Proposition 14 Let $1 \leq p<\infty$, and $w$ and $\omega$ be weight functions on $\mathbb{R}^{d}$. The dual space of $A_{\alpha, p}^{w, \omega}\left(\mathbb{R}^{d}\right)$ is isomorphic to $L_{w^{-1}}^{\infty}\left(\mathbb{R}^{d}\right) \times L_{\omega^{-1}}^{p^{\prime}}\left(\mathbb{R}^{d}\right) / K$ where $\frac{1}{p}+\frac{1}{p^{\prime}}=1$.

\section{Competing interests}

The authors declare that they have no competing interests.

\section{Authors' contributions}

All authors contributed equally to the writing of this paper. All authors read and approved the final manuscript.

Received: 13 October 2014 Accepted: 24 February 2015 Published online: 06 March 2015

\section{References}

1. Feichtinger, HG, Gürkanlı, AT: On a family of weighted convolution algebras. Int. J. Math. Sci. 13, 517-526 (1990)

2. Reiter, H: Classical Harmonic Analysis and Locally Compact Group. Oxford University Press, Oxford (1968)

3. Namias, $\mathrm{V}$ : The fractional order of Fourier transform and its application in quantum mechanics. IMA J. Appl. Math. 25, 241-265 (1980)

4. Ozaktas, HM, Kutay, MA, Zalevsky, Z: The Fractional Fourier Transform with Applications in Optics and Signal Processing. Wiley, Chichester (2001)

5. Almeida, LB: The fractional Fourier transform and time-frequency representations. IEEE Trans. Signal Process. 42 3084-3091 (1994)

6. Zayed, Al: On the relationship between the Fourier and fractional Fourier transforms. IEEE Signal Process. Lett. 3, 310-311 (1996) 
7. Almeida, LB: Product and convolution theorems for the fractional Fourier transform. IEEE Signal Process. Lett. 4(1), 15-17 (1997)

8. Zayed, Al: Fractional Fourier transform of generalized function. Integral Transforms Spec. Funct. 7, 299-312 (1998)

9. Bultheel, A, Martinez, H: A shattered survey of the fractional Fourier transform. Report TW337, Department of Computer Science, K.U. Leuven (2002)

10. Sahin, A, Ozaktas, HM, Mendlovic, D: Optical implementations of two-dimensional fractional Fourier transforms and linear canonical transforms with arbitrary parameters. Appl. Opt. 37(11), 2130-2141 (1998)

11. Sharma, VD: Operational calculus on generalized two-dimensional fractional Fourier transform. Int. J. Eng. Innov. Technol. 3(2), 253-256 (2013)

12. Fischer, RH, Gürkanlı, AT, Liu, TS: On a family of weighted spaces. Math. Slovaca 46(1), $71-82$ (1996)

13. Singh, AK, Saxena, R: On convolution and product theorems for FRFT. Wirel. Pers. Commun. 65, 189-201 (2012)

14. Zayed, Al: A convolution and product theorem for the fractional Fourier transform. IEEE Signal Process. Lett. 5, 101-103 (1998)

15. Liu, T, Rooij, AV: Sums and intersections of normed linear spaces. Math. Nachr. 42, 29-42 (1969)

Submit your manuscript to a SpringerOpen ${ }^{\circ}$ journal and benefit from:

- Convenient online submission

- Rigorous peer review

- Immediate publication on acceptance

- Open access: articles freely available online

- High visibility within the field

Retaining the copyright to your article

Submit your next manuscript at $\boldsymbol{s p r i n g e r o p e n . c o m ~}$ 